\title{
Rente ermöglicht Integration
}

\section{Doris Brühlmeier-Rosenthal}

Dr. med., Fachärztin für Psychiatrie und Psychotherapie, Mitglied FMH

Herr Dummermuth wirft mir vor, mein Artikel basiere auf Subjektivität, Wunschprojektionen und Behauptungen. Als meines Wissens bisher Einzige habe ich jedoch im November 2016 die Mühe auf mich genommen, eine Nachzählung IV-betroffener Patienten in psychiatrischen Praxen vorzunehmen. Die Zahlen sind in der Online-Ausgabe der Schweizerischen Ärztezeitung einsehbar ${ }^{1}$. Ich hoffe, dass ich nicht mehr lange gegen den sozialen Tod der IV-Opfer anschreiben muss und bald wieder in Ruhe meiner geliebten Berufsarbeit nachgehen kann.

\section{Abweisung macht krank}

Als erstes möchte ich Herrn Dummermuth und seinem Kanton gratulieren, dass nur 10 von 261 Patienten mit aufgehobener IV Rente zwischen 2009 bis 2013 zu Sozialhilfeempfängern wurden. Das ist zweifelsohne eine sehr gute Leistung. Auch der Eingliederungserfolg von 65 Prozent ist beachtlich! Der Haken dabei ist allerdings, dass beim Schwyzer Kollektiv nur IV-Berechtigte dabei sind. Die primär von der IV abgewiesenen Menschen (2014: 425 im Kanton Schwyz, Tausende schweizweit) und die beim Verwaltungsgericht hängigen Fälle (2014: 140 im Kanton Schwyz, einige Tausend schweizweit) sind nicht gezählt, weder im Hinblick auf Sozialhilfeabhängigkeit noch auf Erwerbstätigkeit, Gesundheitszustand oder Krankheitskosten. Nicht alle Abgewiesenen sind gesund, und sie werden es durch die Ablehnung erst recht nicht.

Die Personen, die - teils nach polydisziplinären Gutachten - zu 38 oder 39 Prozent arbeitsunfähig, also aus Sicht der IV für gesund erklärt sind, sind häufig krank und werden nach Abweisung durch die IV noch kränker. Ebenso verschlechtert sich der Gesundheitszustand jener Patientinnen und Patienten, die in Rekursverfahren stecken. Alle diese Personen müssten ebenfalls gezählt werden. Viele von ihnen gelangen in unsere Praxen. Auch wenn im Kanton Schwyz nur zehn Personen mit aufgehobener IV-Rente zu Sozialhilfeempfängern wurden, sind es zehn zu viel: Sie brauchen Wiedergutmachung und eine unkomplizierte IV-Rentenzusprache.

Bei meinem nachgezählten Kollektiv handelt es sich ausschliesslich um Patienten psychiatrischer Praxen der Kantone Aargau und Zürich. Das heisst, dass viele Personen dabei sind, deren Rente von der IV abgelehnt wurde oder die in Rekursverfahren stecken, ebenso wie Patienten mit Teil- und Vollrente und solche mit aufgehobener Rente. Das für mich Überraschendste war der hohe Anteil von IV-Rentenbezügerinnen und -bezügern, die im ersten oder zweiten Arbeitsmarkt (teil-)erwerbstätig sind (36-48\% psychisch kranke IVRentner), sowie die weitestgehende Erwerbslosigkeit derjenigen, die von der IV abgewiesen wurden, die auf den Rentenentscheid warten oder deren IV Rente aufgehoben wurde. Der grosse Anteil teilerwerbstätiger IVRentnerinnen und -Rentner (36 Prozent der Vollrentner, 48 Prozent der Teilrentner) ist jedoch nichts Neues. Das war schon so, bevor das Schlagwort «Eingliederung vor Rente» aufkam. Die Jahre bis zur allfälligen Rentenzusprache und nach der Ablehnung einer Rente dürften für die Krankenkassen die teuersten sein. Allein schon Rentenrevisionen bringen Notfallkonsultationen und Rezidive mit sich.

\section{Sozialhilfeabhängigkeit}

Sozialhilfeempfänger wird nur, wer sich anmeldet, wer sein allfällig Erspartes weitgehend aufgebraucht hat und wer keinen Mann beziehungsweise keine Frau hat, die genug verdient. Ich erlaube mir, aus Paul Hallers Juramareili [1] folgende Zeilen einzuflechten:

Mutter: 's goht nümme ohni Hilf, mer müend a d'Gmeind Mareili: Und wenn eus gueti Lüüt wänd öppis geh; So wämmer froh sii. Aber ned uf Gmeind! Und $a b$ der Gmeind go ässe: zähmol lieber: Eläigge luege, lieber Hunger ha.

Sowohl männliche wie vor allem auch weibliche $\mathrm{Pa}$ tientinnen sind mir bekannt, die sozialhilfeberechtigt wären, aber "Juramareili-Blut» in sich haben und den für sie so schweren Gang nicht auf sich nehmen können. Hinzu kommt, dass vielen bewusst ist, dass Sozialhilfe keine Gabe ist, sondern quasi ein zinsfreies Darlehen, das bei wirtschaftlicher Besserstellung zurückerstattet werden muss. Die Dunkelziffer der Sozialhilfeberechtigten, die auf einen Bezug verzichten, ist nicht bekannt. 


\section{Integration dank Rente oder Integration vor Rente?}

Meine Nachzählung und meine Erfahrung in der Praxis zeigen, dass viele psychisch Kranke fatal auf Druck und Überforderung reagieren [2]. Teure Integrationsbemühungen der IV scheitern, weil der Druck infolge der zeitlichen Begrenzung der Integrationsprogramme und Arbeitsversuche zu gross ist. Zu rasche Steigerungen des Arbeitspensums können viel zerstören. So ist es nicht verwunderlich, dass die Integration ausserhalb der IV sehr erfolgreich ist. Oft erlebe ich «Integration dank Rente»: Die Zusprache einer Teil- oder Vollrente und Anerkennung der bestehenden Krankheit bringt so viel Erleichterung, dass Teilerwerbstätigkeit möglich wird. So kommt es auch, dass viele Patienten dank fairen Arbeitgebern und Job Coaches der Erwerbsausfallversicherungen, die prima Arbeit leisten, ihre Stelle halten können, manchmal in angepassten Pensen. Psychische Gesundheit heisst tätig zu sein und Beziehung leben zu können, das sagte schon Sigmund Freud. So bringt die Befreiung dank einer IV-Rentenzusprache oft Erwerbstätigkeit mit sich. Druck hingegen zerstört sie, insbesondere bei seelisch Kranken.

\section{Gut investiertes Geld}

2016 konnte die IV bei der AHV 823 Millionen Franken Schulden zurückzahlen [4], was 40000 Jahresrenten entspricht. Die IV ist also ein prosperierendes Unternehmen geworden, mit einem grossen Gewinn ähnlich einer Bank. Eine durchschnittliche Jahresrente beträgt knapp 20000 Franken pro Jahr. Für diese 20000 Franken gibt es weniger Krankheitskosten, weniger Unglück, weniger soziales Elend, teils für ganze Familien, grosse Chancen kleiner Erwerbstätigkeit. In der Geldsprache nennt man das "gut investiertes Geld». Die Zahl der Neurenten blieb trotz der Bevölkerungszunahme von einer halben Million seit 2010 stabil das heisst, sie nahm kräftig ab gemessen an der Bevölkerung. Die Opfer aber geraten in grosse Not und brauchen Behandlung in unseren Praxen.

\section{Änderungsvorschläge}

Dr. med. Doris Brühlmeier-

Rosenthal

Fachärztin für Psychiatrie

und Psychotherapie

Uitikonerstrasse 4

CH-8952 Schlieren

doris.bruehlmeier[at]

gmail.com

Herrn Dummermuth bitte ich, folgende Anliegen zu unterstützen und eventuell mitzutragen:

- Das Bundesgericht soll seine Rechtssprechung ändern. Als meines Wissens einziges Land in Eu- ropa ist es in der Schweiz juristisch möglich, bei Schmerzpatienten und anderen Kranken die IVRente aufzuheben [3]. Dies muss rückgängig gemacht werden durch dasselbe Bundesgericht, Wiedergutmachung muss ermöglicht werden.

- Das Parlament soll den Spardruck gegenüber der IV beenden und die Schulden gegenüber der AHV erlassen. Die Zahl der Renten darf steigen, so wie auch die Bevölkerungszahl steigt.

- Die IV-Stellen sollen nein sagen zu Rentenkürzungen und Rentenstopps, Entscheidungen rascher treffen und Rekurse vermeiden. Sie sollen die Patienten nicht unter krankmachenden Druck setzen, sondern sie ernst nehmen und "in dubio pro reo» entscheiden (denn als Angeklagte fühlen sich viele Patienten). Auch die Ablehnung von Kosten orthopädischer Schuhe, die plötzlich nicht mehr bezahlt werden, macht krank, ebenso wie ein ungerechtfertigter Rentenentscheid. Entscheide gegen den ärztlichen Rat können lebensgefährdend sein.

- Die Gutachtenden sind gebeten, mit den behandelnden Ärztinnen und Ärzten Kontakt aufzunehmen, bevor Entscheidungen über Patienten gefällt werden. Die Empfehlung der behandelnden Ärzte soll zu 70 Prozent angerechnet werden.

- Wir praktizierenden Ärzte und Psychologen stellen ein grösstmögliches Zahlenkollektiv zusammen. Gemeinsam mit dem Verein Ethik und Medizin Schweiz haben wir einen online-Blitzfragebogen entwickelt [5], mit dem alle IV-betroffenen Patienten gemeldet werden können. Wir hoffen auf Tausende von Meldungen.

- Herrn Dr. Christoph Blocher haben wir schon gebeten, die Kampagne gegen «Scheininvalide» offiziell zu beenden. Die definitive Zusage steht noch aus, ist aber nicht ausgeschlossen

Sind dies gangbare Möglichkeiten, die Lage unserer Kranken zu verbessern, auch ausserhalb des Kantons Schwyz?

Literatur

1 Paul Haller: Juramareili, Neuausgabe Werke, Baden Verlag 2007.

2 Vgl. auch Hell Daniel (2007): Die Bedeutung der IV für psychisch Kranke, SAEZ 2007 88(24)1053/4.

3 Humanrights.ch (04.07.2017): Schmerzpatienten/-innen und psychisch Kranke vor dem Bundesgericht weitgehend chancenlos (Michael E. Meier). https://www.humanrights.ch/de/menschenrechte-schweiz/inneres/gruppen/behinderte/ausschlussschmerzpatienten-iv
4 www.bsv.admin.ch/finanzen der Invalidenversicherung

5 http://varifo.ch/rentenlupe.htm 